\title{
BIBLIOGRAFÍA Y VIDEOGRAFÍA
}

Carlos Gutiérrez Lozano*

Esta recopilación sigue el orden cronológico de las actividades académicas y de investigación de Carlos de la Isla, con exclusión de los libros escritos o compilados por él, que se citan primero para evitar numerosas repeticiones. Para cada título se considera todo lo relacionado con él, cuando lo hubiera, en el siguiente orden: publicación impresa, publicación digital, videograbación, podcast, reimpresión.

\section{Libros}

(1) De la perplejidad a la utopía, 1998, México, Ediciones Coyoacán/ITAM, 242 pp.

(2) De esclavitudes y libertades. Ensayos de ética, educación y política, 2006, México, ITAM/Miguel Ángel Porrúa, 300 pp.

\section{Compilador}

(3) Ética y empresa, 2000, México, ITAM/USEM/FCE, 287 pp.

* Departamento Académico de Estudios Generales, ITAM. 
(4) "Reflexión en plural sobre un camino que ilumine la persona en la educación superior", Revista del Instituto Tecnológico Autónomo de México 1 (1973), pp. 4-13.

- http://generales.itam.mx/sites/default/files/reflexion_en_plural.pdf

- Reimpreso como "De la educación oficial conservadora a la utopía de la imaginación”, Estudios ITAM 37 (1994), pp. 77-84.

- http://biblioteca.itam.mx/estudios/estudio/letras37/notas1/sec_1.html

- Reimpreso en (1), pp. 31-48.

- Reimpreso como "Hacia una educación del hombre humano", en (2), pp. 33-49.

(5) "Breve reflexión sobre los estudios generales en la universidad", Opción 1 (1980), <http://opcion.itam.mx/?p=587>.

(6) "Momentos y límites de la ciencia", presentado en el Seminario sobre el Valor de la Ciencia, organizado por el Centro Universitario de Cultura, octubre de 1981.

- Impreso en (1), pp. 171-184.

(7) “¿Educación para la libertad o para el sometimiento?”, Estudios ITAM 3 (1985), pp. 31-44.

- http://biblioteca.itam.mx/estudios/estudio/estudio03/sec_5.html

- Reimpreso en (1), pp. 61-72.

- Reimpreso en (2), pp. 77-88.

(8) "Relación entre los problemas nacionales y los problemas educacionales", leído en la Junta de Facultad del ITAM, primavera de 1985.

- Impreso en (1), pp. 81-88.

(9) "Responsabilidad social y universidad", intervención en el Seminario de Educación celebrado en El Colegio de México, otoño de 1986.

- http://generales.itam.mx/sites/default/files/responsabilidad_ social_y_univ.pdf 
- Podcast: http:/generales.itam.mx/sites/default/files/responsabilidad_social.mp3

- Impreso en (1), pp. 73-80.

(10) "En torno a las dimensiones reales del capitalismo. Comentario al libro Desarrollo e ideología de Manuel Cazadero", Estudios ITAM 10 (1987), pp. 105-110.

- http://biblioteca.itam.mx/estudios/estudio/estudio10/sec_22.html

- Reimpreso en (1), pp. 211-216.

(11) "De la educación de la memoria al desarrollo de la imaginación", en Javier Beristain et al. (comps.), México al filo del año 2000, 1989, México, ITAM, pp. 27-36.

(12) "La universidad que se piensa a sí misma", en La misión de la universidad, 1990, México, ITAM, pp. 47-51.

- Coloquio La Misión de la Universidad, Querétaro, 11-13 de mayo de 1989 (videograbación), México, ITAM, Departamento de Estudios Generales, 1989, 6 videocasetes Beta, 720 minutos.

(13) "Educación y democracia”, ponencia en la mesa redonda del Seminario sobre Educación organizado por el INI, abril de 1989.

- Impreso en (1), pp. 89-96.

(14) “El problema de la educación para la libertad”, Estudios ITAM 21 (1991), pp. 71-79.

- http://biblioteca.itam.mx/estudios/estudio/letras21/coloq1/sec_1.html

- Reimpreso como "Educación para la libertad" en (1), pp. 97-106.

(15) “La universidad: conciencia crítica”, Estudios ITAM 25, 1991, pp. 69-76.

-http://biblioteca.itam.mx/estudios/estudio/letras25/texto4/sec_1.html

- Podcast: <http://generales.itam.mx/sites/default/files/universidad_ conciencia_critica.mp3 $>$.

- Reimpreso en (1), pp. 107-114. 
(16) Conferencia organizada por Carlos de la Isla (grabación sonora), México, ITAM, 1991, 1 casete, 70 min.

(17) “El futuro de la educación superior en México” (videograbación), México, ITAM, 1992, 1 DVD, 82 min.

(18) "La legitimidad de algunos valores universales", Estudios ITAM 32 (1993), pp. 25-32.

- http://biblioteca.itam.mx/estudios/estudio/letras32/textos3/ sec_1.html

- Reimpreso en (1), pp. 195-202.

(19) "La revolución del pensamiento", leído en la Biblioteca Manuel Gómez Morín para la presentación del libro Cuando por la raza habla el espíritu, Manuel Gómez Morín, Rector de la UNAM (1933-1934), 1995, México, Centro Cultural Manuel Gómez Morín/Jus, invierno de 1995.

- "Cuando por la raza habla el espíritu. Manuel Gómez Morín, Rector de la UNAM, 1933-1934”(videograbación), México, ITAM/ UNAM/Centro de Estudios sobre la Universidad/ Centro Cultural Manuel Gómez Morín, 1994, 3 DVD, 265 min.

- Impreso en (1), pp. 125-130.

(20) "Reflexiones sobre la educación para la invención del futuro", Estudios ITAM 39/40 (1994/5), pp. 199-210.

- http://biblioteca.itam.mx/estudios/estudio/letras39-40/texto16/ sec_1.html

- Reimpreso en (1), pp. 49-60.

(21) “Introducción”, Estudios ITAM 39/40 (1994/1995), pp. 9-14. - http://biblioteca.itam.mx/estudios/estudio/letras39-40/texto02/ sec_1.html

(22) “Descartes: el buscador de evidencias”, Estudios ITAM 47 (1996), pp. 87-92.

- http://biblioteca.itam.mx/estudios/47-59/47/CarlosdelaIsla DescarteselBuscadorde.pdf

- Reimpreso en (1), pp. 203-210. 
(23) "Presentación: De la perplejidad a la utopía”, Estudios ITAM 45/46 (1996), pp. 7-12.

- http://biblioteca.itam.mx/estudios/estudio/letras45-46/texto01/ sec_1.html

- Reimpreso en (1), pp. 25-30.

(24) "En torno a Cien años de presencia y ausencia social cristiana 1891-1991", Estudios ITAM 34 (1996), pp. 77-83.

-http://biblioteca.itam.mx/estudios/estudio/letras34/notas1/sec_1.html

- Reimpreso en (1), pp. 217-224.

(25) “Comentarios sobre Educación liberal de Rodolfo Vázquez”, Isonomía 6 (1997), pp. 183-190.

-Reimpreso en (1), pp. 185-194.

(26) Ceremonia del nombramiento de Profesor Emérito al Dr. Carlos de la Isla Veraza (videograbación). México, ITAM, 1997, 1 DVD, 89 min.

(27) "Agradecimiento", Estudios ITAM 54 (1998), pp. 122-123.

- http://biblioteca.itam.mx/estudios/47-59/54/CarlosddelaIsla Agradecimiento.pdf

(28) "Es necesaria una nueva actitud ética en el mundo", impreso en (1), pp. 133-152.

- Reimpreso en (3), pp. 5-15.

- Reimpreso con modificaciones en (2), pp. 11-19.

(29) "El pontificado de Juan Pablo II y la historia contemporánea" (videograbación), México, ITAM, Departamento Académico de Estudios Generales, 1998, 1 videocasete, 115 min.

(30) Presentación del libro De la perplejidad a la utopía de Carlos de la Isla (videograbación), México, ITAM, 1998, 1 videocasete, 75 min.

(31) Ceremonia de entrega del Doctorado Honoris Causa a don Alberto Baillères (videograbación), México, ITAM/Grupo BAL/GNP, 1999, 1 DVD, 120 min., + 1 supl., 31 pp. 
(32) Presentación del libro Liderazgo, valores y cultura organizacional (videograbación), México, ITAM, Departamento Académico de Estudios Generales, Departamento Académico de Administración, 1999, 1 videocasete, $100 \mathrm{~min}$.

(33) Semana de la Ciudad de México, 22-24 de noviembre de 1999, (videograbación), México, ITAM, Departamento Académico de Estudios Generales, 1999, 4 videocasetes, 400 min.

(34) "Libertad", en Isidro H. Cisneros et al. (comps.), Léxico de la política, 2000, México, FCE, pp. 399-406, con bibliografía.

- Reimpreso en (1), pp. 153-170 sin la bibliografía.

- Reimpreso en (2), pp. 215-232 sin la bibliografía.

(35) "Ética de la empresa", en Gloria Robles y Carlos Alcérreca (eds.), Administración: Un enfoque interdisciplinario, 2000, México, Pearson Educación de México, pp. 393-417.

- Reimpreso como "Ética y empresa" sin las preguntas para la discusión, la bibliografía y los casos en (2), pp. 149-176.

(36) “Entre mito y realidad”, Estudios ITAM 64/65 (2001), pp. 232-236. - http://biblioteca.itam.mx/estudios/60-89/64-65/CarlosdelaIsla Entremitoyrealidad.pdf

- Reimpreso en (2), pp. 283-287.

(37) "De las sombras a la luz o el mito de la caverna", Estudios ITAM 66 (2003), pp. 9-20.

- http://biblioteca.itam.mx/estudios/60-89/66/CarlosdelaIsla Delassombrasalaluzo.pdf

- Reimpreso en (2), pp. 65-75.

(38) "La educación como vida, arte, poesía, libertad y creación", Estudios ITAM 67 (2003), pp. 110-119.

- http://biblioteca.itam.mx/estudios/60-89/67/CarlosdelaIsla Laeducacioncomovidaarte.pdf 
- Presentación de las obras completas del Dr. Joaquín Xirau (videograbación), México, ITAM, 2002, 1 videocasete, 83 min.

- Reimpreso en (2), pp. 273-282.

(39) "Reinauguración de la Biblioteca del Campus Río Hondo", Estudios ITAM 67 (2003), pp. 142-148.

- http://biblioteca.itam.mx/estudios/60-89/67/Reinauguracion delaBibliotecadelcampus.pdf

(40) "Claves de la complejidad: Pensar la educación y pensar el pensamiento" (videograbación), México, ITAM, 2003, 1 videocasete, $92 \mathrm{~min}$.

(41) “Ética y universidad”, Estudios ITAM 69 (2004), pp. 7-18.

- http://biblioteca.itam.mx/estudios/60-89/69/CarlosdelaIsla Eticayuniversidad.pdf

- Podcast: http://generales.itam.mx/sites/default/files/etica_y_ universidad_0.mp3

- "Ética y universidad: reflexiones sobre ética y universidad" (videograbación), México, ITAM, 2003, 1 videocasete, 34 min.

- Reimpreso en (2), pp. 21-31.

(42) "Reflexiones sobre el método dialógico", Estudios ITAM 70 (2004), pp. 7-19.

- http://biblioteca.itam.mx/estudios/60-89/70/CarlosdelaIsla Reflexionessobreel.pdf

- "Reflexiones sobre el método dialógico" (videograbación), México, ITAM, 2003, 1 videocasete, $33 \mathrm{~min}$.

- Podcast: http://generales.itam.mx/sites/default/files/etica_y_ universidad_0.mp3

- Reimpreso en (2), pp. 101-111.

(43) "Homenaje a don Ernesto Garzón Valdés", en Manuel Atienza, El derecho como argumentación, 2004, México, Fontamara, pp. 19-23. - "Ernesto Garzón Valdés, Ética, filosofía de derecho y política" (videograbación), México, ITAM, 2003, 1 videocasete, 153 min. 
(44) “Homenaje a la Dra. Milagros Mier”, Estudios ITAM 70 (2004), pp. 154-156.

- http://biblioteca.itam.mx/estudios/60-89/70/CarlosdelaIsla HomenajealaDra.pdf

(45) "Publicidad y ética", Estudios ITAM 74 (2005), pp. 7-19.

- http://biblioteca.itam.mx/estudios/60-89/74/CarlosdelaIsla Publicidadyetica.pdf

- Reimpreso en (2), pp. 187-197.

(46) "Una perspectiva académica sobre el desafuero" (videograbación), México, ITAM, Departamento Académico de Estudios Generales, 2005, 1 videocasete, $117 \mathrm{~min}$.

(47) “Un acercamiento a la educación del hombre humano", Estudios ITAM 76 (2006), pp. 121-133.

- http://biblioteca.itam.mx/estudios/60-89/76/CarlosdelaIsla Unacercamientoala.pdf

- "Un acercamiento a la educación del hombre humano" (videograbación), México, ITAM, Departamento de Estudios Generales, 2005, 1 videocasete, $c a .40 \mathrm{~min}$.

- Reimpreso en (2), pp. 51-63.

(48) "Estudios Generales: Universidad dentro del tecnológico", Estudios ITAM 78 (2006), pp. 101-105.

- http://biblioteca.itam.mx/estudios/60-89/78/CarlosdelaIsla Universidaddentrodel.pdf

- "Viejos recuerdos" (videograbación), México, ITAM, 2006, 1 videocasete, $90 \mathrm{~min}$.

(49) “El concepto de hombre y la ética", Estudios ITAM 79 (2006), pp. 133-146.

- http://biblioteca.itam.mx/estudios/60-89/79/CarlosdelaIsla Elconceptodehombre.pdf

- Reimpreso en (2), pp. 137-148. 
(50) Ceremonia conmemorativa del 60 aniversario del ITAM (videograbación), México, ITAM, Dirección de Planeación y Desarrollo, 2006, 1 DVD, 91 min.

(51) Presentación del libro De esclavitudes y libertades (videograbación), México, ITAM, Departamento Académico de Estudios Generales e Internacionales, 2006, 1 DVD, 82 min.

(52) "Problemas sociales y retos demográficos del agro" (videograbación), México, ITAM, Departamento Académico de Estudios Generales, 2006, 1 videocasete, $110 \mathrm{~min}$.

(53) “José Manuel Orozco Garibay, Persona y comunidad. De valores y no valores. Axiología mínima”, Estudios ITAM 87 (2008), pp. 167-171.

- http://biblioteca.itam.mx/estudios/60-89/87/CarlosdelaIsla JoseManuelOrozco.pdf

- Presentación del libro Persona y comunidad. De valores y no valores: axiología mínima de José Manuel Orozco Garibay (videograbación), México, ITAM, Departamento Académico de Estudios Generales, 2006, 1 videocasete, $72 \mathrm{~min}$.

(54) “La crisis ética ENRON-Arthur Andersen” (videograbación), México, ITAM, Departamento Académico de Estudios Generales, 2008, 1 DVD, 95 min.

(55) Homenaje a Alberto Sauret y presentación del libro Textos atorrantes (videograbación), México, ITAM, 2008, 1 DVD, 80 min.

(56) “Solo la RSE y una actitud ética puede salvar a México”, 2008, eBiblioteca de la USEM, <http://www.usem.org.mx/ebiblioteca/index. php? $\bmod =$ ebiblioteca\&id=149>.

(57) Comida y presentación del Departamento Académico de Estudios Generales (videograbación), México, ITAM, Departamento Académico de Estudios Generales, 2009, 1 DVD, 118 min. 
(58) Descripción y contenido de las materias de Estudios Generales (videograbación), México, ITAM, Departamento Académico de Estudios Generales, 2009, 1 DVD, 122 min.

(59) “Urge en México una abundante riqueza limpia”, eBiblioteca de la USEM, <http://www.usem.org.mx/archivos/contenido/articulo interes/urge_abundante_riqueza_limpia.pdf $>$.

(60) “Mundo, pensamiento y lenguaje", Estudios ITAM 93 (2010), pp. 147-158.

- http://biblioteca.itam.mx/estudios/90-99/93/carlosdelaisla mundopensamientoylenguaje.pdf

(61) "Universidad, ética y responsabilidad social", en Carlos Heredia et al. (comps.), La flecha al aire. Homenaje a Javier Beristain: Ensayos sobre economía, desarrollo e innovación educativa, 2010, México, ITAM/CIDE/Miguel Ángel Porrúa, pp. 339-352.

- Presentación del libro Laflecha al aire: Homenaje a Javier Beristain (videograbación), México, ITAM, Departamento de Relaciones Públicas, 2010, 1 DVD, 110 min.

(62) “Alberto Sauret in memoriam”, Estudios ITAM 94 (2010), pp. 159-164.

- http://biblioteca.itam.mx/estudios/90-99/94/carlosdelaisla albertosauretinmemoriam.pdf

(63) Seminario de Compromiso Social, 27 de enero de 2010 (videograbación), México, ITAM/ Dirección de Planeación y Desarrollo/ Asociación de Exalumnos del ITAM, 2010, 3 DVD, 210 min.

(64) "El primer paso a El México que queremos", en Alejandro Sanders, Mariana Barragán y Aldo García (coords.), El México que queremos: Propuesta ciudadana, 2015, México, Telefónica Movistar/ Club Piso 51/UnAM/ITAM/Grupo Financiero Banorte/ Asociación Mexicana de Dirección en Recursos Humanos/ Fundación UNAM/ Centro de la OCDE en México para América Latina, pp. 104-105. 
(65) "Breve reflexión sobre la universidad y sus grandes enemigos”, primera parte, El Supuesto 341, 11 de marzo de 2015, pp. 1 y 3; segunda parte, El Obelisco, 2015, pp. 12-13.

(66) "A la democracia moderna no le queda mucha vida", El Economista, 18 de diciembre de 2016.

\section{$* * *$}

\section{Sobre Carlos de la Isla Veraza}

(1) Luzelena Gutiérrez de Velasco, "Carlos de la Isla: un disidente alado”, Estudios ITAM 54 (1998), pp. 104-107.

- http://biblioteca.itam.mx/estudios/47-59/54/LuzelenaGutierrez deVelascoCarlosdelaisla.pdf

(2) Rodolfo Darío Vázquez Cardozo, "Ética y educación en torno al pensamiento de Carlos de la Isla”, Estudios ITAM 83 (2007), pp. 141-151.

- http://biblioteca.itam.mx/estudios/60-89/83/RodolfoVazquez Eticayeducacionen.pdf

(3) José Rafael González Díaz, "Entrevista a Carlos de la Isla: el rostro humano en la educación contemporánea”, Estudios ITAM 101 (2010), pp. 111-134.

- http://biblioteca.itam.mx/estudios/100-110/101/RafaelGonzalez DiazEntrevistaacarlos delaisla.pdf

(4) Luzelena Gutiérrez de Velasco, "Carta abierta a don Carlos de la Isla”, Estudios ITAM 94 (2010), pp. 169-174.

- http://biblioteca.itam.mx/estudios/90-99/94/luzelenagutierrez develascounacartaabierta.pdf

(5) Alberto Sauret, "Carta abierta a don Carlos de la Isla", Estudios ITAM 94 (2010), pp. 153-159.

- http://biblioteca.itam.mx/estudios/60-89/83/AlbertoSauret CartaabiertaaDonCarlosde.pdf 
CITAM Derechos Reservados.

La reproducción total o parcial de este artículo se podrá hacer si el ITAM otorga la autorización previamente por escrito. 